\section{DIGITAL \\ SIMULATION \\ FOR \\ AUTOMOBILE \\ MANEUVERS}

Keywords: digital simulation, feedback engineering, mechanical engineering, transportation, vehicle dynamics
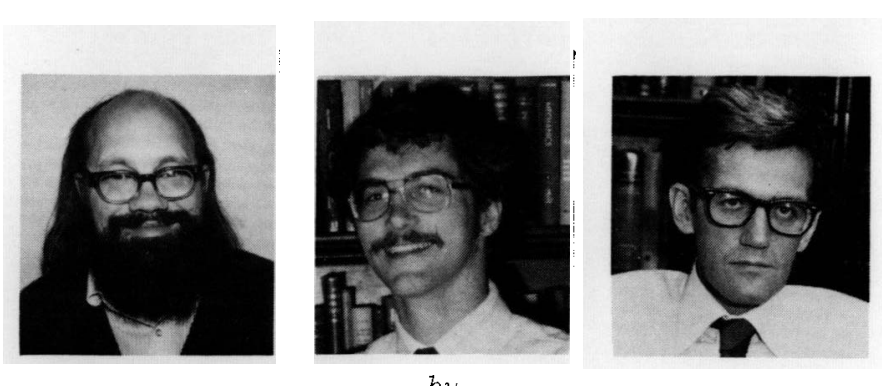

by

W. Riley Garrott,* Douglas L. Wilson, and Richard A. Scott

Department of Mechanical Engineering

and Applied Mechanics

University of Michigan

Ann Arbor, Michigan 48109

W. RILEY GARROTT received a BS in engineering science from Tufts University in 1972 and a $P h D$ in applied mechanics from the California Institute of Technology in 1977. From 1977 to 1979 , he was a postdoctoral Fellow at the University of Michigan, where he conducted research in vehicle dynamics and driver performance, an interest he has kept up during a stint with the Ford Motor Company in 1979 and at his current position.

DOUGLAS L. WILSON obtained a BS in engineering science in 1976 and master's degrees in applied mechanics and bioengineering in 1979 , all from the University of Michigan. His research interests include biomechanics and vehicle dynamics. He is currently working on his PhD in applied mechanics.

RICHARD A. SCOTT received a BSc in experimental physics in 1957 and an MSc in mathematical science in 1959 from the National University of Ireland. In 1964 he obtained a PhD degree in engineering science from the California Institute of Technology. After spending three additional years at Caltech as a lecturer and a postdoctoral fellow, he joined the University of Michigan in 1967, where he is currently a professor of mechanical engineering and applied mechanics. His recent research interests have been in elastic wave propagation, nonlinear and random vibration, and vehicle dynamics.

\section{ABSTRACT}

A new all-digital simulation of automobile handling allows severe maneuvers involving braking or acceleration and cornering. A novel feature is the incorporation of closed-loop control based on a mathematical model of the human driver. The program is modular and well-documented. The model includes provisions for nonlinear tire and suspension forces and moments; it also allows the user to switch off the noninearities and to include an antilock brake system.

* Currently at the Vehicle Research and Test Center, East Liberty, Onio 43319

\section{INTRODUCTION}

In another work ${ }^{10}$ we have described a five-degreeof-freedom digital simulation of straight-1ine automobile maneuvers. Here we report on the recent development of a general all-digital simulation of open- and closed- loop automobile maneuvers includeng severe turning and braking. Bohn ${ }^{1}$ reviewed early work in this area thoroughly in 1973. Of particulare note is McHenry and DeLeys, 9 development in 1968 of a mathematical model for open-loop dynamics. This model operated on a hybrid computer at the Bendix Research Laboratories; it later evolved into the National Highway Traffic Safety Administration's Hybrid Computer Vehicle Handling Program (HVHP). ${ }^{3} 11$ Bohn and Keenan have described the HVHP model, which is now set up in an updated form at the Johns Hopkins University. Jindra? describes the original HVHP model.

The basic goals and achievements of our simulation were:

(1) Including models of the human driver to allow study of closed-1oop maneuvers

(2) An all-digital form that does not require a hybrid facility and is therefore readily available to more potential users

(3) Extensive modularization of the program to facilitate future extensions (for example, the program is structured so that changes in the tire-force module do not require extensive changes in the suspension module)

\section{LIST OF SYMBOLS}

$u, v$ Velocity components of the sprung mass in the and $x$ and $y$ directions, respectively, where $x, y$, and $z$ are the fixed axes of the body

$x, y$ Inertial coordinates $\delta$ Angular displacement of the steering wheel 
(4) Capability of handling the nonlinearities of severe maneuvers

(5) Provision for an antilock system (which can be switched off) in which front and rear brakeline pressures are apportioned to avoid lock-up of the wheels

(6) Extensive documentation to facilitate use and alteration of the model

(7) An interactive mode for hands-on experimentation. Items 1 and 2 , in particular, represent a considerable advance over previous models.

The simulation consists of two main parts: a vehicle model called IDSFC and a general-purpose driver module called DRIVER. Four subroutines handle the interfacing between the driver module and the vehicle model. These subroutines can be readily altered to allow the driver module to be used with different vehicle models.

The vehicle model IDSFC has the following degrees of freedom:

- Sprung mass: three translational and three rotationa1

- Front unsprung masses: two wheel hops, two wheel spins, two wheel rotations about the king-pins, and one steering connecting-rod displacement (ro reduce costs, the steering system is handled statically)

- Rear unsprung masses: in the case of a solid rear axle, one deflection of the rear suspension, one rear-axle roll, and two wheel spins; in the case of independent rear suspensions, two rear-suspension deflections and two wheel spins.

The mathematical representation of the vehicle involves 30 first-order nonlinear differential equations and approximately 250 algebraic equations. The program contains 30 subroutines; both singleprecision and double-precision versions are available.

The simulation has the following capabilities:

(1) It allows straight-line braking and acceleration, cornering without braking or acceleration, and cornering with braking or acceleration.

(2) It retains nonlinear kinematic terms, allowing the user to study severe maneuvers. Switches (i.e., the specification of certain program parameters) activate the nonlinearities allowing them to be excluded easily from the simulation of maneuvers in which they are not significant, thereby decreasing running costs. The user can also employ these switches to study the effects of various nonlinearities. The models of tire and suspension forces and moments include the principal nonlinearities.

(3) It provides two methods for computing tire sideforces: the APL-CALSPAN model, which is based on curves fitted to the measured data, and a Partial Data Deck model which uses the measured data directly.

(4) It provides an antilock feature activated by a model level switch.
(5) It allows both solid rear axle and independent rear suspensions.

(6) It provides for front-wheel drive, rear-wheel drive, and four-wheel drive. The wheel-spin differential equations are treated algebraically to reduce costs.10

(7) It permits separate braking at each wheel.

(8) It includes an interactive capability, which is activated by a model-level switch.

The general-purposes driver module DRIVER provides the control input to the vehicle model. DIRVER's main characteristics are as follows:

(1) The driver controls steering, braking, and drive torque inputs to the vehicle model.

(2) It includes five preprogrammed open-1oop maneuvers:

(a) Sinusoidal steer with trapezoidal braking

(b) Trapezoidal steer with trapezoidal braking

(c) Double trapezoidal steer (input consisting of two sequential trapezoidal steers of opposite signs) with trapezoidal braking; this simulates a lane change

(d) Trapezoidal steering with a sinusoidal perturbation combined with trapezoidal braking

(e) Sinusoidal steering, with no braking, in which the frequency increases linearly with time; this maneuver is designed to produce frequency response information and has no physical interpretation.

(3) It accepts any open-10op maneuver supplied by the user in the tabular form or specified by a user-supplied subroutine.

(4) It can operate in a closed-10op mode to follow a desired path. In this mode four control strategies are available:

(a) A crossover model for a straight-line path (a crossover model includes both feedback and feedforward loops)

(b) A crossover model for an arbitrary path

(c) A preview-predictor model which uses a geometric predictor (preview-predictor models assume that the driver mentally calculates a predicted path and a desired path and then generates control commands based on differences between the two)

(d) A preview-predictor model which uses a three-degree-of-freedom vehicle model as a predictor.

(5) It permits a mixed-mode operation combining open- and closed-loop control.

(6) It permits an obstacle avoidance strategy using either of the preview-predictor models.

\section{SIMULATION VALIDATION}

We validated the current simulation model (IDSFC) for open-loop maneuvers by extensive comparisons of its outputs with corresponding outputs from the APL 
hybrid simulation, which was validated by comparisons with earlier field tests done in connection with the work of McHenry and DeLeys. ${ }^{9}$ Further corroboration was provided by Chiang and Star, ${ }^{4}$ who developed for the Ford Motor Company an all-digital simulation for open-loop maneuvers using essentially the APL mathematical model. Ford Motor Company performed extensive field tests to validate this simulation and obtained good agreement with experimental data.

In validating the IDSFC mode1, we made a comprehensive set of comparison runs involving many variables, only a few of which are given here. The first set involves a straight-line braking maneuver. The vehicle parameters, supplied by APL, correspond to a 1971 Ford Mustang. The initial speed was taken to be $50 \mathrm{mph}(22.35 \mathrm{~m} / \mathrm{s})$, and a ramp brakeline pressure was applied. The rise time of the ramp was taken to be 0.1 second, and three different values of the peak pressure were used. We ran the simulation in the fully nonlinear double-precision mode with the APLCALSPAN tire model.

Figures 1,2 , and 3 give results for a peak brakeline pressure of $475 \mathrm{psi}(3.275 \mathrm{MPa})$. Figure 1 gives the forward velocity $u$ as a function of time; it shows excellent agreement between the two simulations, with a maximum difference of approximately $0.34 \mathrm{mph}$ $(0.15 \mathrm{~m} / \mathrm{s})$. (Note that the APL simulation shuts off at a higher speed than does IDSFC). Figure 2 shows the lateral velocity $v$ versus time, while Figure 3 gives the vehicle trajectory in inertial coordiates, i.e., $Y$ versus $X$. Since the maneuver is a straight line, $v$ and $y$ should be zero. IDSFC makes them zero, whereas the APL results show small deviations from the ideal values.

Figures 4 through 6 show results for peak brakeline pressure of $550 \mathrm{psi}(3.792 \mathrm{MPa})$. The maximum difference between the two predictions of the forward velocity is about $1.16 \mathrm{mph}(0.51 \mathrm{~m} / \mathrm{s})$. The APL results again show minor differences in $v$ and $y$ from their ideal zero values, but IDSFC does not.

The second set of comparison maneuvers involved steering without braking. The vehicle is initially traveling at $50 \mathrm{mph}(22.35 \mathrm{~m} / \mathrm{s})$ along a straight line; then, a trapezoidal steering pulse is applied without braking. In such a pulse the steering wheel angle is increased linearly, held constant for a while, and then decreased to zero, causing the vehicle to change direction. The rise time, dwell time, and fall time of the pulse are one second, and the peak value of the change in steering-wheel angle is 60 .

Figure 7 shows the vehicle trajectory $Y$ versus $X$. Here again, we see excellent agreement between the simulations. Figures 8 and 9 present forward velocity $u$ and transverse velocity $v$ of the sprung mass versus time, respectively. The IDSFC output in Figure 8 is physically more reasonable since it predicts a slowing down of the vehicle. The differences between the two simulations in absolute terms, though, are quite small. Figure 9 shows good agreement for the $v$-versus-time results.

Figures 10 through 12 again describe a trapezoidal steer with the rise time, dwell time, and fall time each increased to two seconds. The same overall trends are apparent, and the agreement between the two simulations is very good on the whole.
Validation of the driver models must await field tests.

\section{SIMULATION SIZE AND RUNNING COSTS}

The single-precision version of IDSFC plus DRIVER occupies approximately $500 \mathrm{~K}$ bytes of memory. The program is written in FORTRAN. We made some relative cost studies using the Amdahl 470 system at the University of Michigan.

Table 1, for a moderate cornering and braking maneuver, gives computing costs and selected important outputs for four modes of running IDSFC. The following assumptions (mentioned in the table) lead to linearization of some of the underlying equations:

(1) The distance between the center of gravity of the rear axle and the roll center is taken to be zero.

(2) Angular velocities are so small that their products and squares can be neglected.

(3) The roll and pitch angles are small.

(4) The suspension's vertical deflections are negligible compared to the height of the center of gravity. In our experience with the simulation, this assumption is a poor one and should never be made.

(5) The suspension deflections and their time derivatives are so small that their products, and products of them with angular velocities, can be neglected.

(6) The roll angle $\phi_{R}$ is so small that the following products can be neglected:

$$
\phi_{R} \dot{\phi}_{R}, \quad \phi_{R} \dot{\phi}_{R}^{2}, \quad \phi_{R}^{2}, \quad \phi_{R}^{2} \dot{\phi}_{R}, \quad \dot{\phi}_{R}^{2}, \quad \phi_{R} \ddot{\phi}_{R}
$$

(7) Some minor terms not present in the APL simulation can be neglected.

Tables 2 and 3 give similar information for a severe steering maneuver without braking and a severe steering maneuver with braking.

All the tables show that removing the nonlinear terms from the mathematical model results in a cost saving of about $20 \%$, with only minor losses in accuracy (a maximum of about $5 \%$ ). These results are important since one of our goals was to assess the effects of nonlinearities in the severe regimes. The tables also show that operating in the singleprecision mode cuts costs by $40 \%$, with an accuracy loss of less than $1 \%$. Based on these observations, we recommend the use of the single-precision versions of the programs. The double-precision versions would be preferable if the user has an exceptionally severe maneuver requiring extra accuracy in the computations.

\section{SAMPLE OUTPUT}

Figures 1 through 12 (discussed above) are a good sample of simulated output for open-loop maneuvers. Here, we present some limited results on closed-loop maneuvers. Reference 6 will provide much more extensive results and discussions.

The maneuver in question involves the effect of a wind gust on a vehicle traveling at a constant speed 


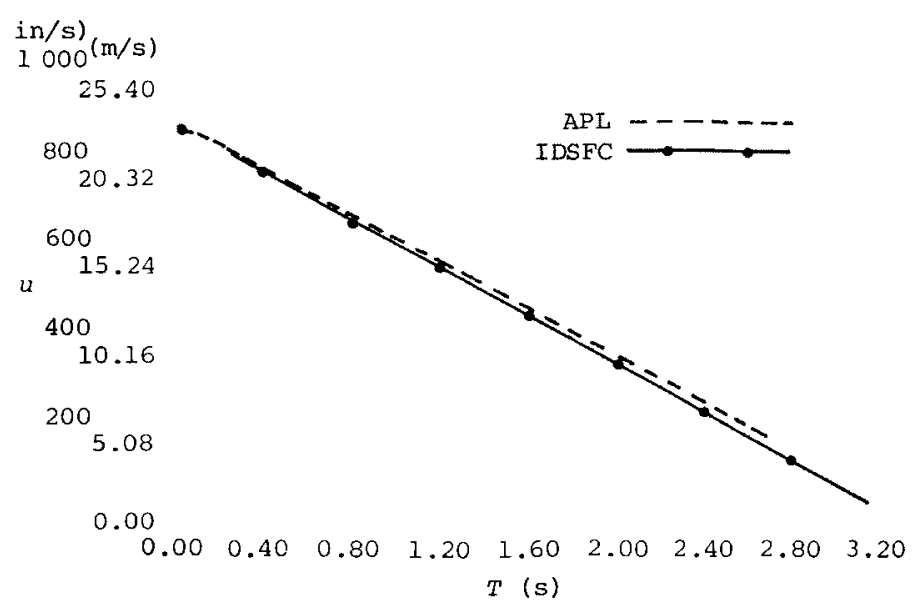

Figure 1 - Forward velocity $u(\mathrm{~m} / \mathrm{s})$ as a function of time $T$ (seconds) for a straight-line braking maneuver with a peak brakeline pressure of $475 \mathrm{psi}(3.275 \mathrm{MPa})$

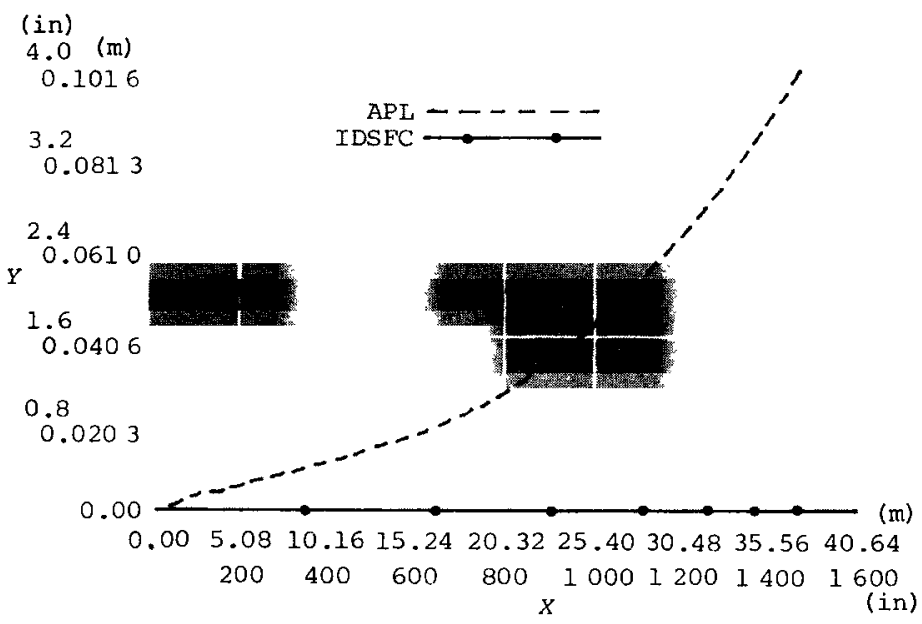

Figure 3 - Vehicle trajectory $Y$ versus $X$ for a straight-line braking maneuver with a peak brakeline pressure of 475 psi (3.275 MPa)

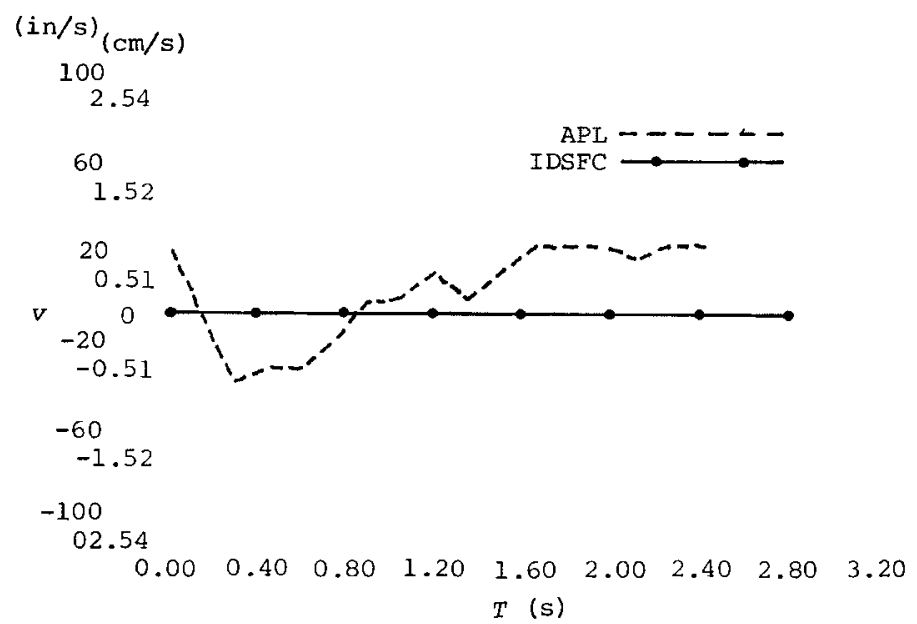

Figure 5 - Lateral velocity $v$ as a function of time $T$ for a straight-line braking maneuver with a peak brakeline pressure of $550 \mathrm{psi}$ (3.79s MPa) $(\mathrm{in} / \mathrm{s})$
$100(\mathrm{~m} / \mathrm{s})$
2.54

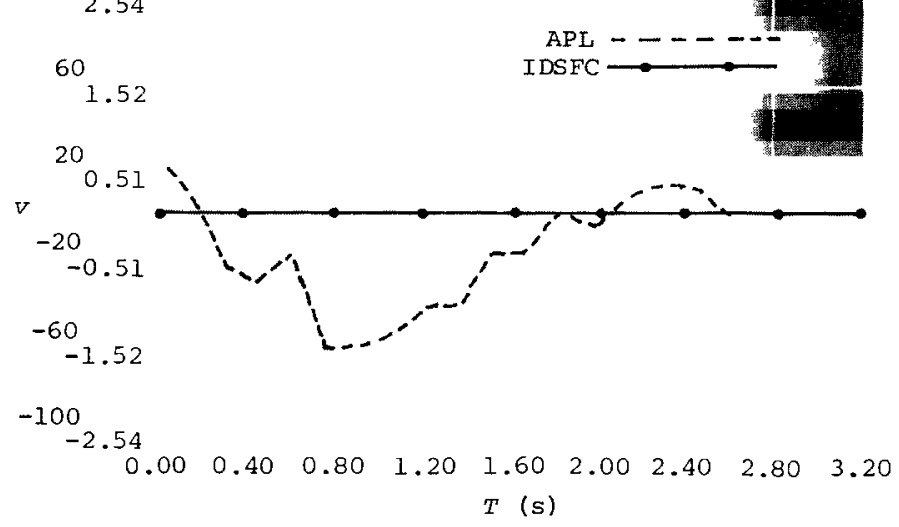

Figure 2 - Lateral velocity $v$ as a function of time $T$ for a straight-line braking maneuver with a peak brakeline pressure of 475 psi (3.275 MPa)

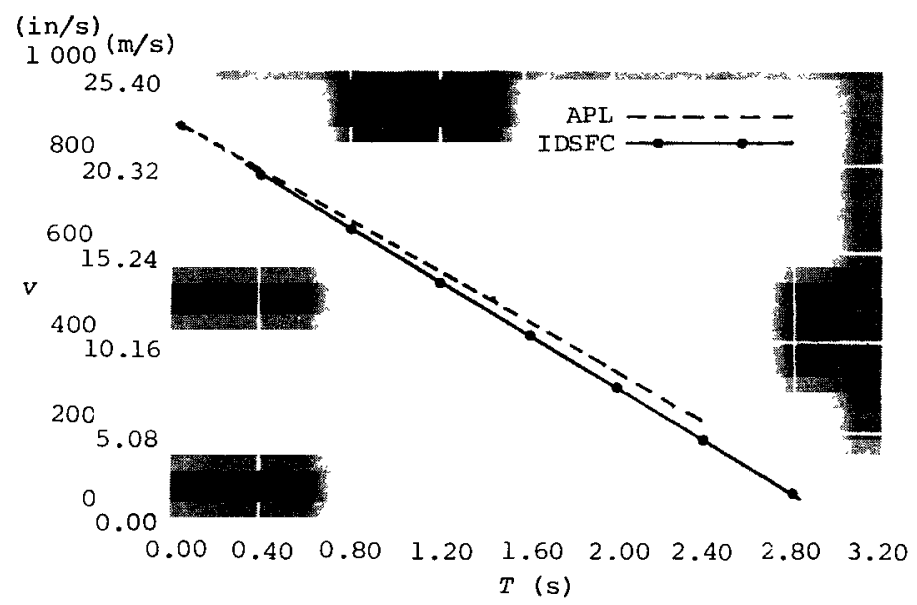

Figure 4 - Forward velocity $u$ as a function of time $T$ for a straight-line braking maneuver with a peak brakeline pressure of $550 \mathrm{psi}$ (3.792 $\mathrm{MPa}$ )

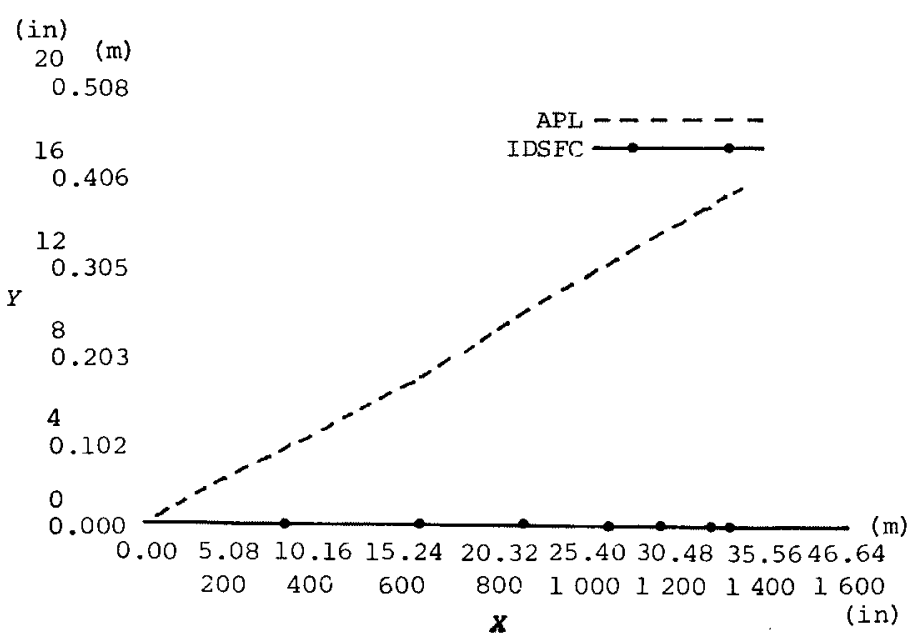

Figure 6 - vehicle trajectory $Y$ versus $X$ for a straight-Iine braking maneuver with a peak brakeline pressure of 550 psi ( $3.792 \mathrm{MPa}$ ) 


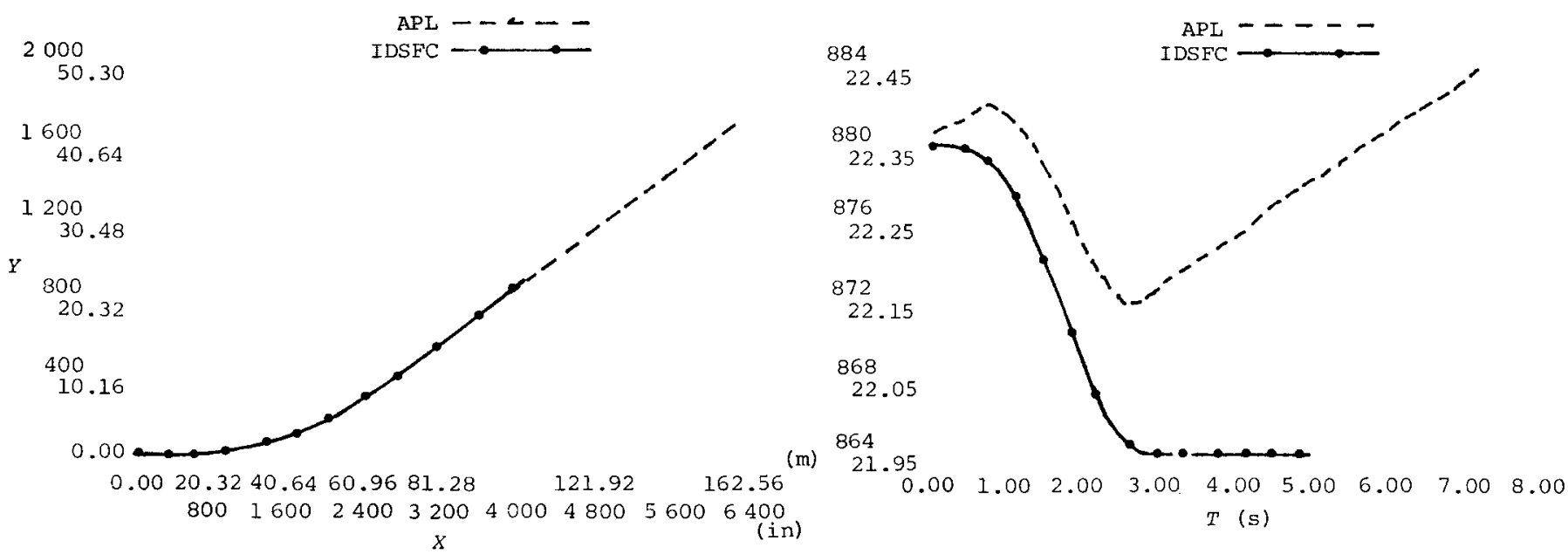

Figure 7 - Veficle trajectory $Y$ versus $X$ for a

Figure 8 - Forward velocity $u$ as a function of time $T$ for a trapezoidal stee

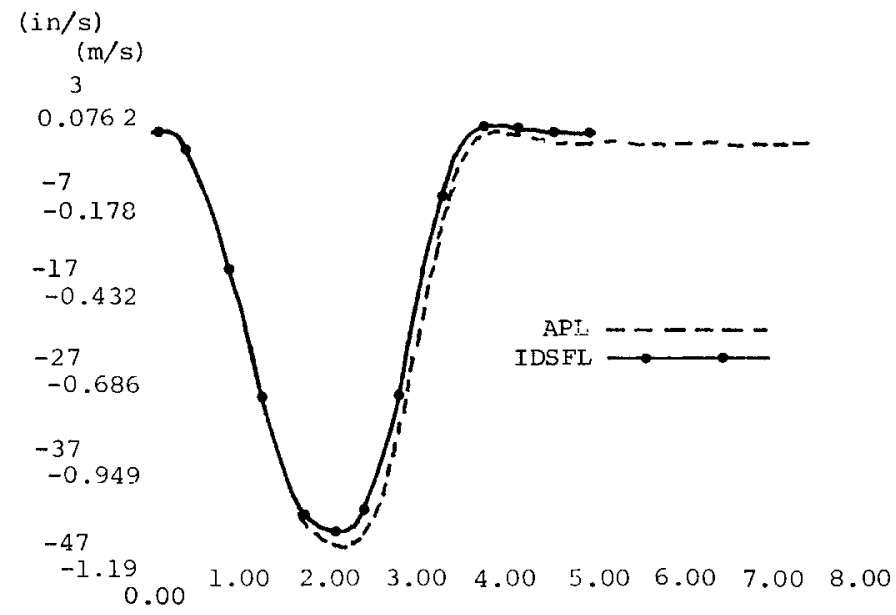

Figure 9 - Lateral velocity $v$ as a function of time $T$ for a trapezoidal steering maneuver

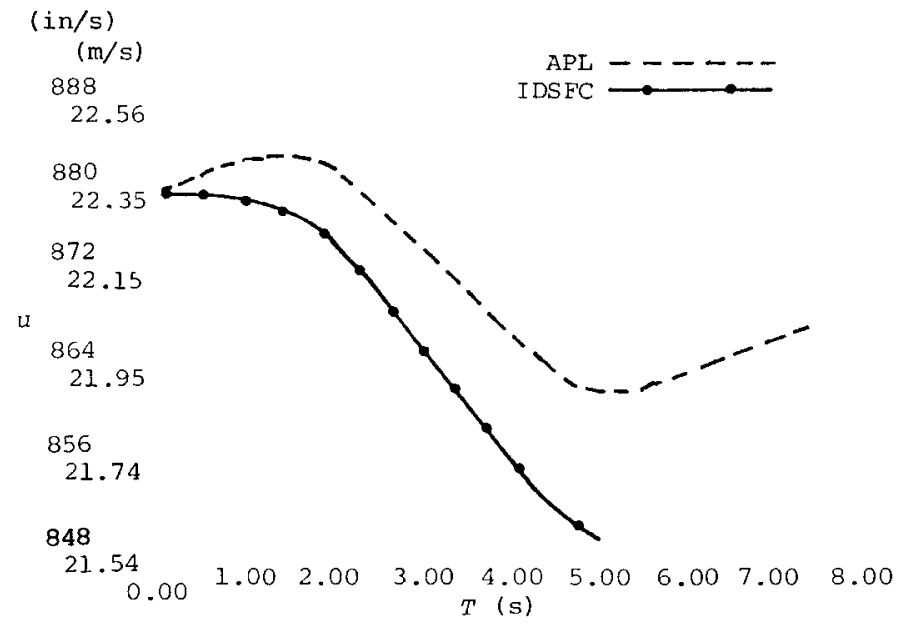

Figure 11 - Forward velocity $u$ as a function of time $T$ for a trapezoidal steering maneuver

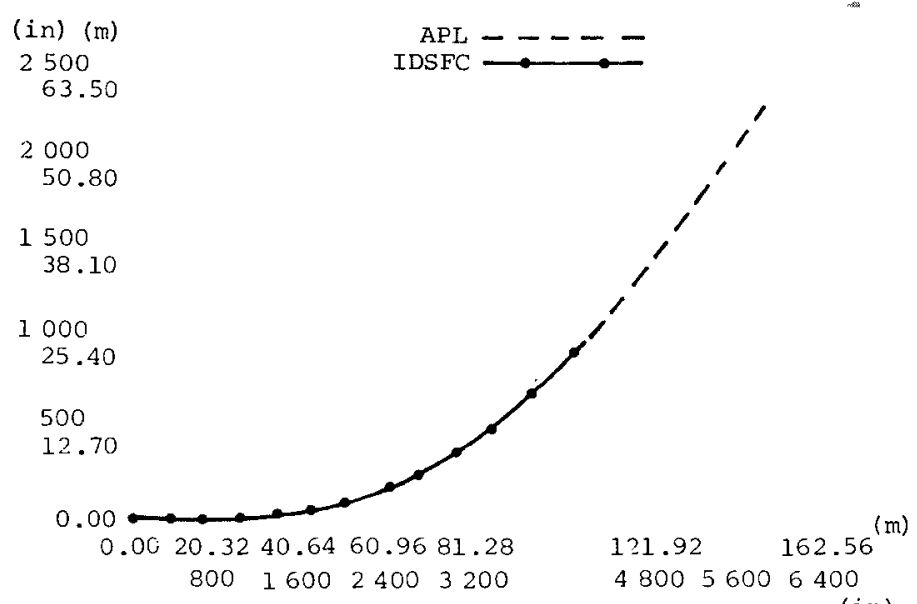

(in)

Figure 10 - vehicle trajectory $Y$ versus $X$ for a trapezoidal steering maneuver

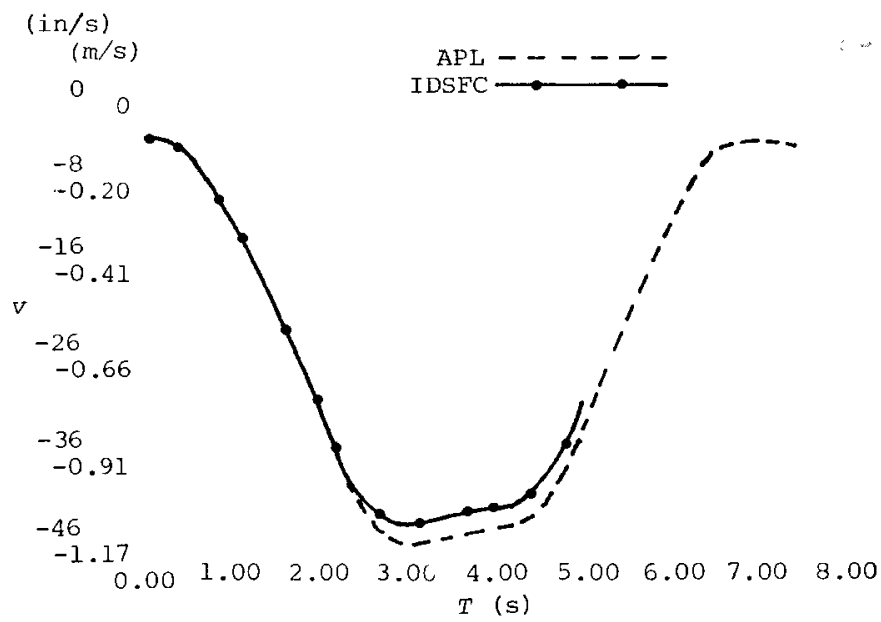

Figure 12 - Lateral velocity $V$ versus time $T$ for a trapezoidal stecring maneuver 
Table 1

Moderate cornering with braking maneuver

(1) The initial speed of the vehicle is $50 \mathrm{mph}(22.35 \mathrm{~m} / \mathrm{s})$.

(2) Ramp braking is applied with a rise time of 0.1 second and a peak hydraulic pressure of 400 psi. (2.758 MPa).

(3) Ramp steering is applied with a rise time of 1 second and a peak value of 43 degrees of the turning angle of the steering wheel.

Double precision

Quantity

Computation
cost per
vehicle-second
CPU time (s)
Maximum
X-acceleration
(gees)
Maximum
Y-acceleration
(gees)
Maximun yaw
rate (deg/s)
Final
$X$-position
Final
$Y$-position
Vehicle
stopping
distance
Vehicle
stopping time
(s)

(s)

(no assumptions except negligible of the suspension)

$-0.6042$

0.1743

6.4319

$44.1481 \mathrm{~m}$

$3.4191 \mathrm{~m}$

$134.6105 \mathrm{in}$

$3.4191 \mathrm{~m}$

144.843 in

3.765 vertical deflection

\section{Double precision}

(al1 assumptions)

Single precision

(no assumptions)

$\$ 1.82$

11.856

$-0.6058$

0.1788

$\$ 1.39$

10.606

$-0.6052$

6.6280

6.4390

$44.1944 \mathrm{~m}$

$44.1134 \mathrm{~m}$

1739.93 in

1736.74 in

$3.5146 \mathrm{~m}$

$3.4168 \mathrm{~m}$

134.5190 in

$138.3717 \mathrm{in}$

$3.4167 \mathrm{~m}$

$4.1945 \mathrm{~m}$

$144.5190 \mathrm{in}$

3.770

3.765

Single precision

(all assumptions except negligible vertical deflection of the suspension)

$\$ 1.12$

8.744

$-0.6058$

6.6268

0.1788

$44.1558 \mathrm{~m}$

1738.41 in

$3.5082 \mathrm{~m}$

$138.1189 \mathrm{in}$

$3.5082 \mathrm{~m}$

144.868 in

3.765

along a straight line $(Y=0)$. The wind gust is simulated by moving the vehicle instantaneously sideways a distance of one foot $(0.3 \mathrm{~m})$. The driver's task is to return the vehicle to the straight-line course $(Y=0)$ while maintaining a constant speed. The initial speed was taken to be $40 \mathrm{mph}(17.88 \mathrm{~m} / \mathrm{s})$ and, as before, the vehicle involved is a 1971 Ford Mustang.

Figure 13 gives the vehicle trajectory $Y$ versus $X$, obtained using the preview-predictor model and the general crossover model. In the predictor process, two methods were employed. In one, a simple stepwise integration technique led to an essentially geometrical method of determining the predicted path. In the second, the predicted path and velocity are generated by solving numerically the differential equations of motion of a separate, three-degree-offreedom vehicle model. This is, of course, a much more accurate procedure, but it is also much more expensive.

A general crossover model is a control theory scheme that allows departures from a straight path. The driver parameters in preview-predicting are those given by Dro11. ${ }^{8}$ In the absence of field data for such modeling, the driver's neuromuscular time lag has been set to zero. For the general crossover model, we obtained the driver parameters by requiring the overall steering-control gain to have a slope of $20 \mathrm{~dB}$ per decade at the crossover frequencies.* For all three driver models, the closedloop system was stable in that it approached the desired state rapidly. Note, though, that the predictor models produce oscillatory behavior, whereas the crossover model has a monotonic behavior, as in an overdamped system.

Figure 14 gives the steering wheel angle $\delta S w$ as a function of time $T$ for the wind gust. In all cases we get a rapid return to the desired zero values. The jerky motion of the steering wheel in the geometric predictor model stems from the nature of the command process. The three-degree-of-freedom predictor produces smoother motion, which is not too surprising in view of its more sophisticated nature.

* The authors are indebted to Calvin Matle of Ford Motor Company for this procedure and for the values obtained using it. 
Tab1e 2

Severe steering maneuver without braking

(1) The initial speed of the vehicle is $55 \mathrm{mph}(24.59 \mathrm{~m} / \mathrm{s})$.

(2) A sinusoidal steer with amplitude of 2 radians at the steering wheel and a period of 1 second is applied.

(3) There is no braking.

(4) The running time is 5 seconds.

\begin{tabular}{|c|c|c|c|c|}
\hline Quantity & $\begin{array}{l}\text { Double precision } \\
\text { (no assumptions) }\end{array}$ & $\begin{array}{l}\text { Double precision } \\
\text { (a1l assumptions } \\
\text { except negligible } \\
\text { vertical deflection } \\
\text { of the suspension) }\end{array}$ & $\begin{array}{l}\text { Single precision } \\
\text { (no assumptions) }\end{array}$ & $\begin{array}{l}\text { Single precision } \\
\text { (all assumptions } \\
\text { except negligible } \\
\text { vertical deflection } \\
\text { of the suspension) }\end{array}$ \\
\hline $\begin{array}{l}\text { Computation } \\
\text { cost per } \\
\text { vehicle second }\end{array}$ & $\$ 2.21$ & $\$ 1.24$ & $\$ 1.34$ & $\$ 1.12$ \\
\hline CPU time (s) & 19.242 & 15.312 & 13.850 & 11.494 \\
\hline $\begin{array}{l}\text { Maximum } \\
X \text {-acceleration } \\
\text { (gees) }\end{array}$ & -0.0214 & -0.0209 & -0.0214 & -0.0208 \\
\hline $\begin{array}{l}\text { Maximum } \\
Y \text {-acceleration } \\
\text { (gees) }\end{array}$ & 0.6648 & 0.6566 & 0.6647 & 0.6565 \\
\hline $\begin{array}{l}\text { Maximum yaw } \\
\text { rate deg/s }\end{array}$ & 19.0602 & -19.0475 & 19.0611 & -19.0461 \\
\hline $\begin{array}{l}\text { Final } \\
X \text {-position }\end{array}$ & $\begin{array}{l}108.7333 \mathrm{~m} \\
4280.8374 \mathrm{in}\end{array}$ & $\begin{array}{c}108.4539 \mathrm{~m} \\
4269.8386 \mathrm{in}\end{array}$ & $\begin{array}{rl} & 108.7766 \mathrm{~m} \\
4 & 282.5430 \mathrm{in}\end{array}$ & $\begin{array}{l}108.4978 \mathrm{~m} \\
4271.5669 \mathrm{in}\end{array}$ \\
\hline $\begin{array}{l}\text { Final } \\
Y \text {-position }\end{array}$ & $\begin{array}{c}37.8025 \mathrm{~m} \\
1488.1869 \mathrm{in}\end{array}$ & $\begin{array}{c}38.0093 \mathrm{~m} \\
1496.4294 \mathrm{in}\end{array}$ & $\begin{array}{c}37.8072 \mathrm{~m} \\
1488.4724 \text { in }\end{array}$ & $\begin{array}{c}38.0167 \mathrm{~m} \\
1496.7200 \mathrm{in}\end{array}$ \\
\hline Final velocity & $22.1991 \mathrm{~m} / \mathrm{s}$ & $22.1453 \mathrm{~m} / \mathrm{s}$ & $22.1766 \mathrm{~m} / \mathrm{s}$ & $22.1238 \mathrm{~m} / \mathrm{s}$ \\
\hline
\end{tabular}

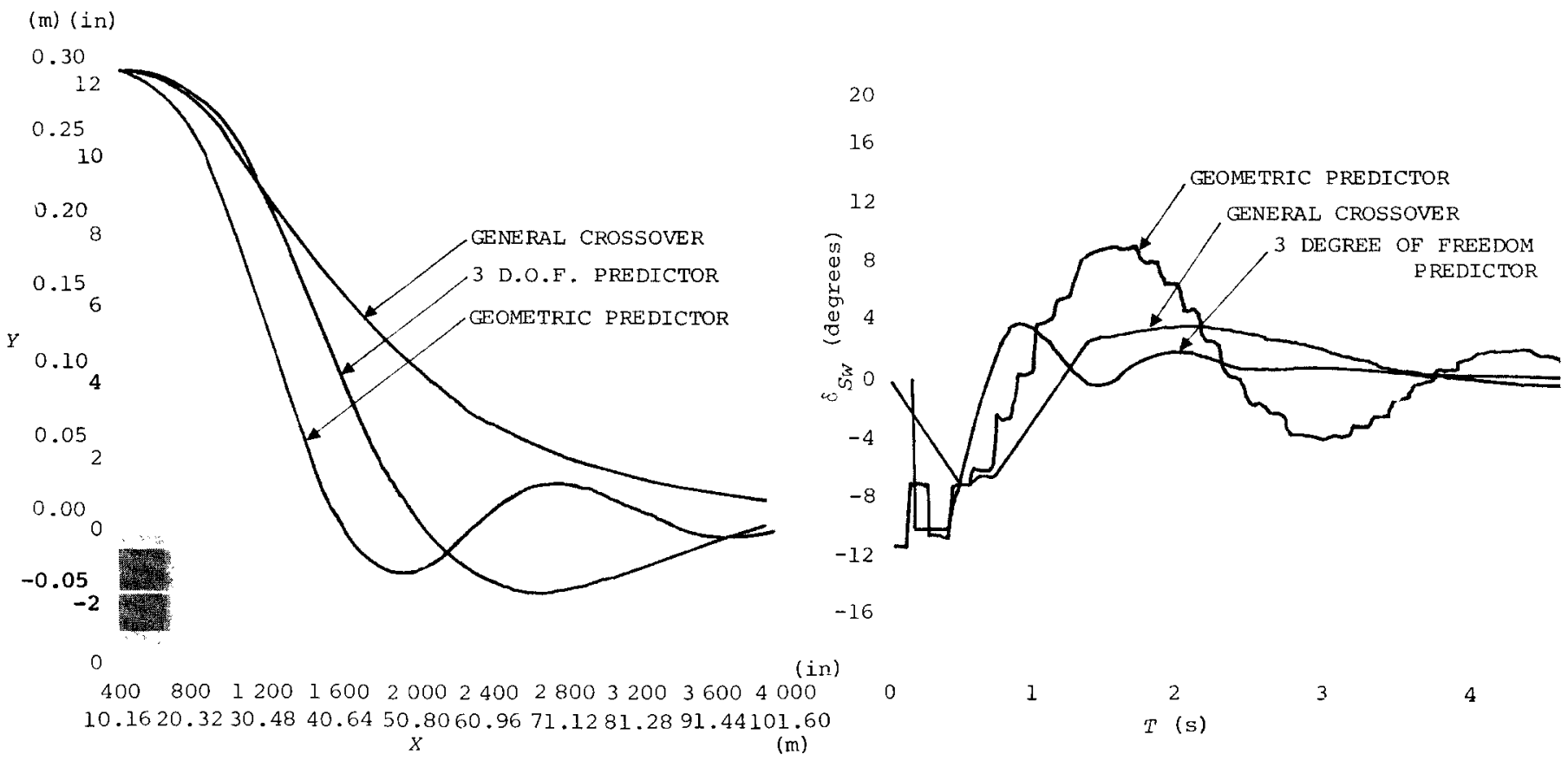

Figure 13 - Vehicle trajectory $Y$ versus $X$ for the wind gust

Figure 14 - Steering wheel angle $\delta_{S W}$ versus time $T$ 
Severe steering with braking maneuver

(1) The initial vehicle speed is $55 \mathrm{mph}(24.59 \mathrm{~m} / \mathrm{s})$.

(2) Ramp braking is applied with a maximum brake-1ine pressure of 480 psi (3.310 MPa) and a rise time of 0.1 second.

(3) A sinusoidal steer is applied with an amplitude of $1 \frac{1}{2}$ radian at the steering wheel and a period of 1 second.

Double precision

Quantity

\begin{abstract}
Computation
cost per

vehicle second

CPU time (s)

Maximum

$X$-acceleration

(gees)
\end{abstract}

Maximum

$Y$-acceleration

(gees)

Maximum yaw

rate $\mathrm{deg} / \mathrm{s}$

Maximum 1ong.

slip of

wheel 1

Maximum long

slip of

wheel 2

Maximum long.

slip of

wheel 3

Maximum long.

slip of

wheel 4

Final

$X$-position

Final

$Y$-position

Vehicle
stopping
distance

Vehicle

stopping time

(s) (all assumptions except negligible vertical deflection at the suspension)

Double precision

(no assumptions)

$\$ 2.38$

14.531

$-0.7351$

0.1729

11.7848

1.00000

0.14498

1.00000

0.14252

$45.4267 \mathrm{~m}$

$1788.4512 \mathrm{in}$

$4.3229 \mathrm{~m}$

169.6257 in

$45.4268 \mathrm{~m}$

$149.038 \mathrm{ft}$

3.540
$\# 1.33$

11.881

$-0.7416$

0.2573

11.7772

1.00000

0.14707

1.00000

0.14924

$45.9155 \mathrm{~m}$

1807.6978 in

$$
4.3085 \mathrm{~m}
$$

$169.6257 \mathrm{in}$

$45.9154 \mathrm{~m}$

$150.641 \mathrm{ft}$

3.575
Single precision

(no assumptions)

$\$ 1.45$

10.437

$-0.7351$

0.2729

11.779

1.00000

0.14498

1.00000

0.14255

$45.3925 \mathrm{~m}$

$1787.1064 \mathrm{in}$

$$
5.3158 \mathrm{~m}
$$

$169.9121 \mathrm{in}$

$45.3726 \mathrm{~m}$

$148.926 \mathrm{ft}$

3.535
8.720

Single precision

(Al1 assumptions except negligible vertical deflection of the suspension)

$\$ 1.18$

$-0.7416$

0.2573

11.7777

1.00000

0.14707

1.00000

0.14924

$45.842 \mathrm{~m}$ 1806.4658 in

$4.3046 \mathrm{~m}$ 169.4707 in

$45.9943 \mathrm{~m}$ $150.539 \mathrm{ft}$

3.575
The result from the crossover model is still smoother, except in the first half-second where the effects of a driver time lag and of limits set on the steering-wheel velocity rate are clearly evident.

Figure 15 shows lateral velocity as a function of time $T$ for the wind gust. All three models predict a rapid response to the zero state, but again the general crossover model leads to a smoother driving process. However, the question of which model most closely reproduces human driving behavior awaits definitive field tests. 


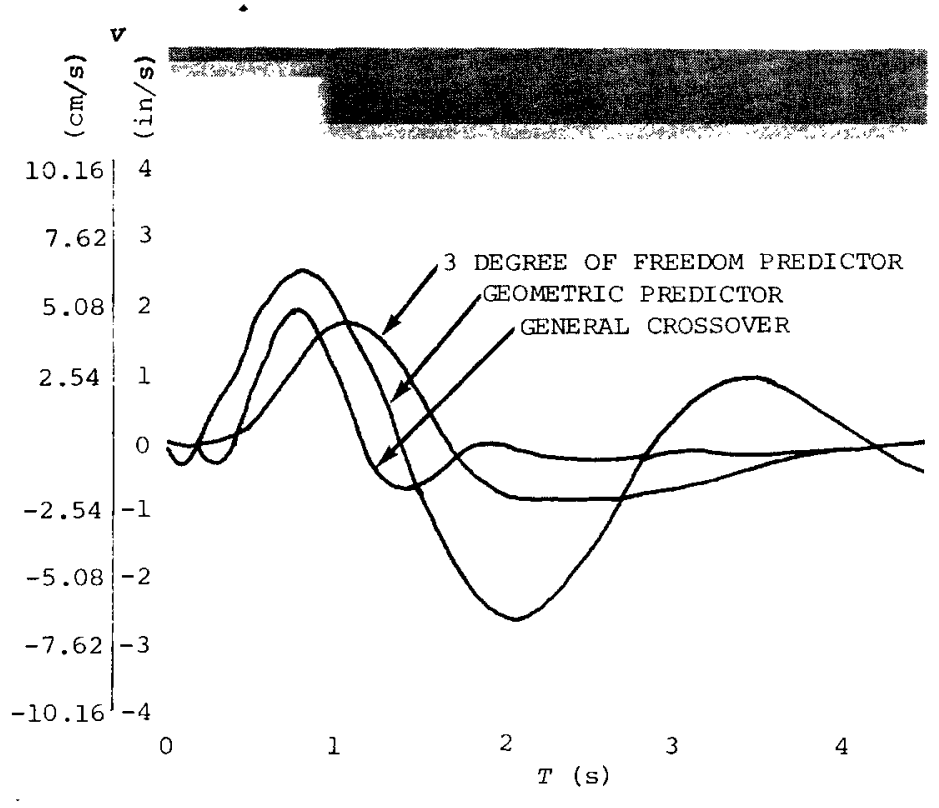

Figure 15 - Lateral velocity $V$ versus time $T$ for the wind gust

\section{REFERENCES}

1 BOHN, P.F.

Modeling and Simulation in Vehicle Handling Research

Proceedings Symposium on Vehicle Safety Research Integration pp. 143-169

Department of Transportation-Highway Safety-820306 May 1973

2 BOHN, P.F. KEENAN, R.J. Improved Hybrid Computer Vehicle Handling Program Report no. CP 049 A Applied Physics Laboratory Johns Hopkins University October 1978

3 Computer similation of Vehicle Handing Department of Transportation-Highway Safety-800789 NATSA Control FH-11-7563

Bendix Research Laboratories Southfield, Michigan September 1972

4 CHIANG, S.L. STARR, D.S.

Using Computer Simulation to Evaluate and Improve Vehicle Handling

Paper no. 780009 Society of Automobile Engineers

5 GARROTT, W.R. SCOTT, R.A.

Improvement of Mathematical Models for Simulation of Vehicle Handing

Technical Manual for the General Simulation: Final Report vol. 7

Department of Transportation-Highway Safety-701715 March 1980
The programs for all the simulations discussed in this paper are available through the National Highway Traffic Safety Administration. Magnetic tape copies can also be obtained at cost from the last author.

Finally, we should note that development of these simulation models is still under way. We are currently modifying IDSFC to include vehicle assymmetries, such as offset payload. The role of these assymmetries will then be assessed in various rightturn and left-turn maneuvers.

\section{ACKNOWLEDGEMENT}

The work reported here was funded by the National Highway Traffic Safety Administration under DOT-IIS-7-01715. The authors are indebted to Joseph Kanianthra, contract technical manager, for his advice and encouragement. Appreciation is also due to Paul Fancher of the Highway Safety Research Institute of the University of Michigan for many helpful discussions and suggestions.

6 GARROTT, W.R. WILSON, D. SCOTT, R.A. Some Studies on Closed-Loop Automobize Maneuvers. Part I: Theory. Part 2: Results International Joumal of Vehicle Systems Design in press

7 JINDRA, F. Mathematical Model of a Four-Wheel Vehicle for the Hybrid Computer

Vehicle Handling Program Department of Transportation-Highway Safety-801-800 1975

8 KROLL, C.V. et al.

A Preview-Predictor Model of Driver Behavior in Emergency Situations

Contract no. CPR-11-3988 Federal Highway

Administration 1970

9 MCHENRY, R.R. DELEYS, N.J .

Vehicle Dynamics in Single Vehicle Accidents-Validation and Extensions of a Computer Simulation Report no. VJ-2251-V-3 Cornell Aeronautical Laboratory, Inc. Cornell University Ithaca, New York December 1968

10 MITCHELl, B. ABRAMS, R. SCOTT, R.A. A Five-Degree-of-Freedom Digital Simulation for Straight-Line Braking/Acceleration Maneuvers Simulation in press

11 Vehicle Hondling: Final Report. II: Technical Report

Department of Transportation-Highway Safety-800282

Bendix Research Laboratories Southfield, Michigan Apri1 1970 